\title{
Pembrolizumab-induced autoimmune Stevens-Johnson syndrome/toxic epidermal necrolysis with myositis and myocarditis in a patient with esophagogastric junction carcinoma: a case report
}

\author{
Jiashun Cao ${ }^{1 \#}$, Qiu Li ${ }^{2 *}$, Xiuyi Zhi ${ }^{1}$, Fan Yang ${ }^{1}$, Weipeng Zhu ${ }^{1}$, Ting Zhou ${ }^{1}$, Xianming Hou ${ }^{1}$, Donghong Chen ${ }^{1}$ \\ ${ }^{1}$ Department of Thoracic Surgery, Beijing Tsinghua Changgung Hospital, School of Clinical Medicine, Tsinghua University, Beijing, China; \\ ${ }^{2}$ Tsinghua University Clinical Research Center, Beijing Tsinghua Changgung Hospital, School of Clinical Medicine, Tsinghua University, Beijing, \\ China \\ \#These authors contributed equally to this work. \\ Correspondence to: Donghong Chen, MD. Department of Thoracic Surgery, Beijing Tsinghua Changgung Hospital, School of Clinical Medicine, \\ Tsinghua University, Beijing 102218, China. Email: cdha01121@btch.edu.cn.
}

\begin{abstract}
Immune checkpoint inhibitors (ICIs) have significantly improved outcomes for cancer patients. With the widespread clinical application of ICIs, their adverse reactions have gradually been recognized. The side effects of ICIs are generally less severe than those of chemotherapy. However, some adverse events with ICIs can be life-threatening. Fatal adverse events require a deep understanding and vigilance. Here, we report the case of a 69-year-old patient with esophagogastric junction carcinoma who experienced multiple immune-related adverse events, including Stevens-Johnson syndrome/toxic epidermal necrolysis, myositis, myocarditis, and liver toxicity, after receiving pembrolizumab. The patient experienced skin rash, bilateral ptosis, limb weakness, and shortness of breath. The symptoms progressed rapidly. Following treatment with methylprednisolone, intravenous immunoglobulin, and plasmapheresis, the patient recovered well. No tracheal intubation or tracheotomy was required owing to the timely and effective treatment. From this case, it can be seen that severe skin rash is an important indication of abnormal immune status and an early warning sign of subsequent multiple-organ involvement. As the most dangerous adverse event, myocarditis is closely related to the patient's prognosis. Severe irAEs appear early, progress rapidly, and involve multiple systems and organs, resulting in a high fatality rate. Early recognition and high-dose corticosteroids are key to successful treatment of such patients.
\end{abstract}

Keywords: Pembrolizumab; Stevens-Johnson syndrome/toxic epidermal necrolysis (SJS/TEN); myositis; myocarditis; case report

Submitted Mar 16, 2021. Accepted for publication May 21, 2021.

doi: $10.21037 /$ tcr-21-470

View this article at: https://dx.doi.org/10.21037/tcr-21-470

\section{Introduction}

Immune checkpoint inhibitors (ICIs) are human-derived monoclonal antibodies that can disrupt tumor immune evasion by blocking the programmed cell death 1
(PD-1)/programmed death-ligand 1 (PD-L1) pathway, thus restoring the anti-cancer effects of immune cells. Several types of ICIs targeting PD-1 or PD-L1 are available worldwide. The use of ICIs has led to breakthroughs in lung cancer treatment and other malignancies (1).

\footnotetext{
^ ORCID: 0000-0002-8150-3958.
} 
With the widespread clinical application of ICIs, their adverse reactions have gradually been recognized. Fatal adverse events require a deep understanding and vigilance. In this report, we described the case of a patient with esophagogastric junction carcinoma in whom multiple immune-related adverse events were induced by pembrolizumab. We present the following article in accordance with the CARE reporting checklist (available at https://dx.doi.org/10.21037/tcr-21-470).

\section{Case presentation}

A 69-year-old man presented at our hospital with a 1-week history of bilateral eyelid drooping after receiving chemotherapy plus immunotherapy as postoperative adjuvant therapy. Two months before this hospital visit, the patient was diagnosed with esophagogastric junction carcinoma and underwent a proximal subtotal gastrectomy in our hospital. The patient's pathologic stage was T3N3M0G3, IVA (American Joint Committee on Cancer, $8^{\text {th }}$ edition). He had no history of rheumatic disease, tuberculosis, or hepatitis.

One month after his operation, the patient was readmitted to the hospital to receive the first cycle of adjuvant chemotherapy plus pembrolizumab. The regimen consisted of oxaliplatin $150 \mathrm{mg}$ one day $1+$ tegafur, gimeracil, and oteracil porassium capsule $60 \mathrm{mg}$ BID on days D1 to 14+ pembrolizumab $200 \mathrm{mg}$ on day 1 . The patient did not complain of any discomfort during his treatment and was discharged from the hospital. 2 weeks after the end of adjuvant treatment, a large rash of flat spots appeared on his chest, which increased in size, merged gradually, and was accompanied by itching and visible blisters (Figure 1A). The involved area was mainly concentrated on the face, neck, anterior chest wall, abdominal wall, and palms, accounting for $28 \%$ of body surface area (face $3 \%$, forequarters $13 \%$, hands $5 \%$, and feet $7 \%$ ). A large number of inflammatory secretions appeared around the palpebral fissure, and were accompanied by blurred vision. Artificial tears were required to keep the patient's eyes moist. Considering the probability of Stevens-Johnson syndrome/toxic epidermal necrolysis (SJS/TEN), which is serious pembrolizumab-induced skin toxicity, using pembrolizumab was discontinued permanently.

Consequently, the $2^{\text {th }}$ cycle of adjuvant therapy was adjusted to chemotherapy alone, after which the patient was discharged home. After that, the erythema began to desquamate and developed into extensive exfoliation of epidermal necrosis. Uneven pigmentation and tender new skin appeared after the exfoliation (Figure 1B). New finger nails gradually replaced the old ones (Figure 1C).

One week before the current hospital visit, bilateral ptosis of the upper eyelids, which was more severe on the right side, appeared, accompanied by diplopia. The patient's symptoms became progressively worse. A sense of limb weakness and shortness of breath appeared simultaneously. After re-admission, blood gas analysis under a $5 \mathrm{~L} / \mathrm{min}$ oxygen inhalation via nasal catheter revealed: partial pressure of oxygen (pO2), $157.3 \mathrm{mmHg}$; partial pressure of carbon dioxide (pCO2) $37.2 \mathrm{mmHg}$; and arterial oxygen saturation $(\mathrm{SaO} 2 \%), 99 \%$. Venous blood test results showed: creatine kinase (CK), 6,016 U/L; CKMB, $128.4 \mathrm{ng} / \mathrm{mL}$; myoglobin (MYO) 3,333 ng/mL; highsensitivity cardiac troponin $\mathrm{T}$ (TnT-hs), $2.83 \mathrm{ng} / \mathrm{mL}$; $\mathrm{N}$-terminal pro-brain natriuretic peptide (NT-proBNP), 3,644 pg/mL; alanine transaminase (ALT), $190.3 \mathrm{U} / \mathrm{L}$; aspartate aminotransferase (AST), $338 \mathrm{U} / \mathrm{L}$; and lactate dehydrogenase (LDH), 1,258 U/L. Anti-Sjgren syndrome A antibody (Anti-SSA) and antinuclear antibody (ANA) were positive. Human leukocyte antigen-DR isotype (HLADR; class II) was $68.49 \%$. Thyroid hormones, adrenal cortisol, creatinine, and urea nitrogen were in the normal range. Thyroglobulin and thyroid peroxidase antibodies were negative. The levels of $\mathrm{B}$ and $\mathrm{T}$ lymphocytes, and natural killer cells in peripheral blood were decreased, while the CD4/CD8 ratio increased to 3.38. Acetylcholine receptor, acetylcholinesterase, titin, ryanodine receptor, and muscle-specific kinase antibodies were all negative. An electrocardiogram (ECG) revealed a first-degree atrioventricular block, frequent ventricular premature, premature ventricular bigeminy, and ST-segment elevation (Figure 2). Echocardiography revealed that the left ventricular ejection fraction (EF) had decreased to $30 \%$. The neostigmine test was negative. Electromyography showed that the sympathetic skin response (SSR) was not elicited in the limbs. The diagnosis was considered as immunotherapy-induced adverse events (irAEs), involving the skin, myocardium, skeletal muscle, and liver.

The patient's symptoms progressed rapidly. Just 5 days after the admission, the patient was still experiencing shortness of breath, despite the delivery of $10 \mathrm{~L} / \mathrm{min}$ oxygen via an oxygen mask, and he was unable to open both eyes completely (Figure 3A). Considering the high probability of requiring short-term mechanical assisted ventilation, he was transferred to the intensive care unit for further treatment. Positive pressure ventilation through a noninvasive ventilator was adopted immediately in the ICU. 

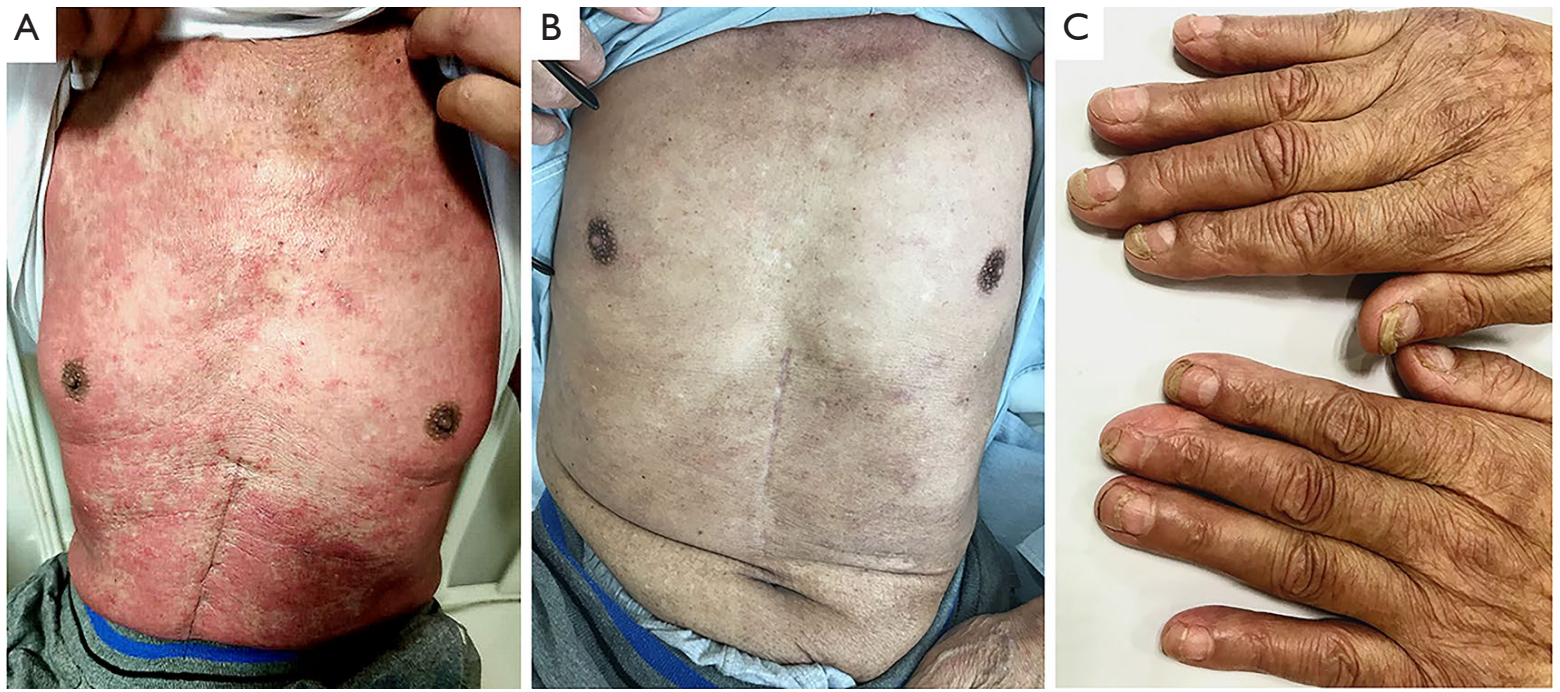

Figure 1 Skin changes during treatment. (A) Skin rash on the chest at onset; (B) exfoliation of the chest was completed after 2 months of treatment; (C) exfoliation of both hands was completed, and new ones gradually replaced old fingernails.
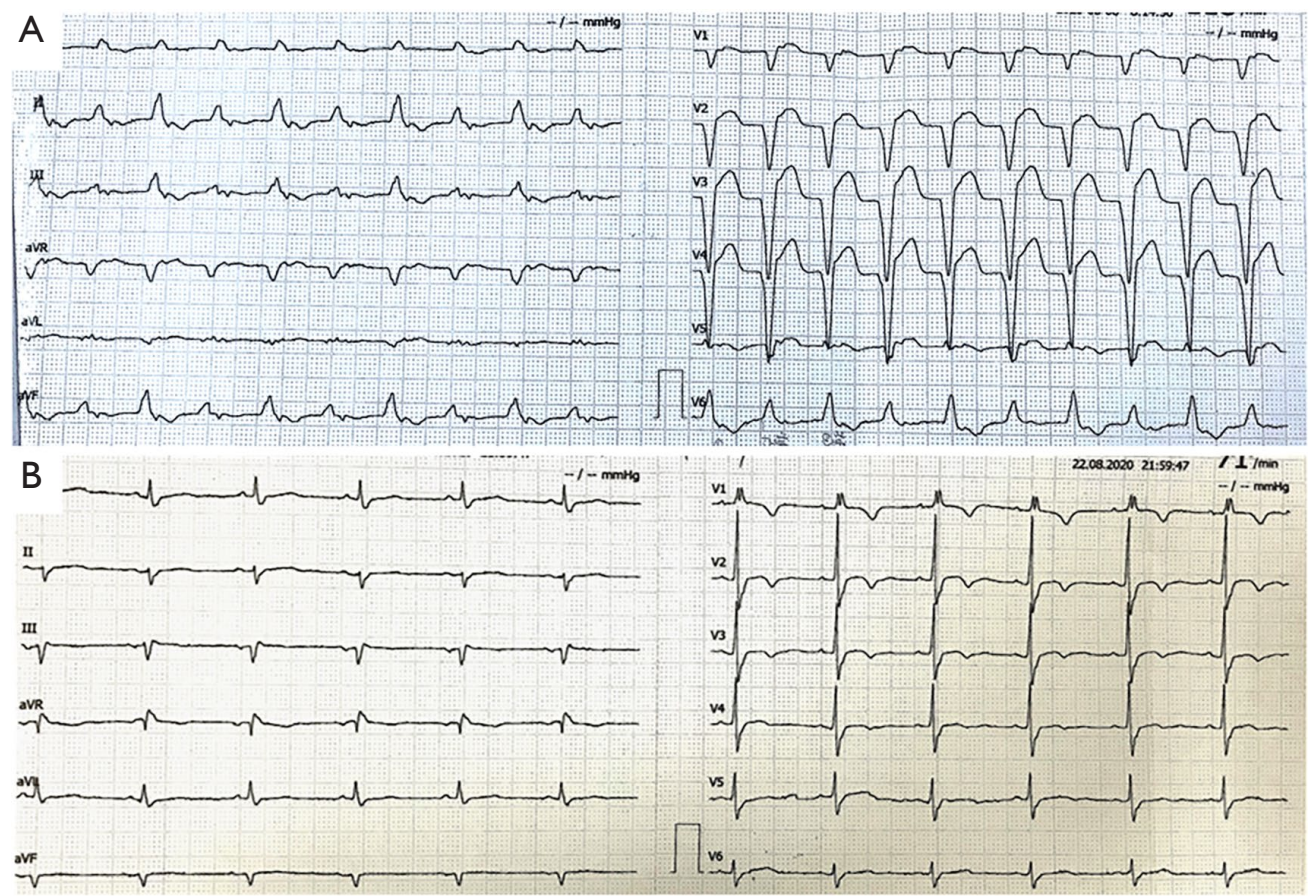

Figure 2 ECG changes during the therapy. (A) ECG at onset; and (B) ECG changes after 2 weeks of treatment. ECG, electrocardiogram.

Liver protection, and antiplatelet therapy were also used. At the same time, treatment with methylprednisolone 500 $\mathrm{mg} \mathrm{Qd}^{*} 3 \mathrm{~d}$ was started. As a result of the treatment, the myocardial enzymes showed a downward trend. The left ventricular end-diastolic diameter increased by $12 \mathrm{~mm}$; the left ventricular posterior wall thickness decreased from $10 \mathrm{~mm}$ to $7 \mathrm{~mm}$; the myocardial edema improved. The dose of methylprednisolone was then adjusted to $250 \mathrm{mg} \mathrm{Qd}$ 3d. Intravenous immunoglobulin (20 g Qd) and plasmapheresis $(2,000 \mathrm{~mL})$ were given simultaneously. The 
symptom of suffocation was gradually relieved. Myocardial enzymes maintained a downward trend. ECG showed that the frequent premature ventricular contractions had disappeared and the PR interval had returned to normal (Figure 2B). The left ventricular EF had increased to $45 \%$. The patient's condition kept improving as the dose of methylprednisolone was gradually reduced.
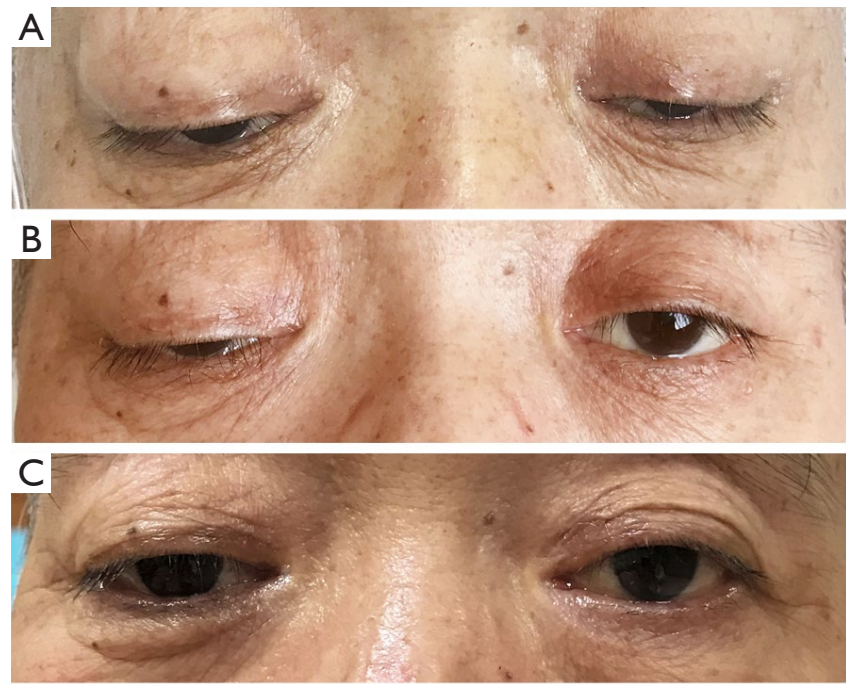

Figure 3 Ocular changes during treatment. (A) Ocular features at onset; (B) ocular features after 1 month of treatment; and (C) ocular features after 2 months of treatment.
From the 10th day of admission to the ICU, the noninvasive ventilatory support was removed intermittently and replaced with high-flow nasal congestion and largevolume atomized oxygen inhalation. The oxygenation index was kept above 400 . On the 17 th day of ICU admission, the ventilator was removed completely, and the patient's symptoms improved significantly (Figure $3 B, C$ ). The methylprednisolone was then adjusted to $30 \mathrm{mg} \mathrm{Qd}$, and the patient was transferred to the general ward (Figure 4). He remained in a stable condition and was discharged from the hospital 1 week later.

All procedures performed in studies involving human participants were in accordance with the ethical standards of the institutional and/or national research committee(s) and with the Helsinki Declaration (as revised in 2013). Written informed consent was obtained from the patient.

\section{Discussion}

A meta-analysis by Wang et al. involving 12,808 patients who received PD-1/PD-L1 inhibitor therapy showed that the overall incidence of irAEs was $26.82 \%$, all grade severe irAEs was $6.10 \%$, and the rate of irAE-associated mortality was $0.17 \%$ (2). The CTLA-4 inhibitor ipilimumab result in a significantly higher incidence of severe enteritis and skin toxicity than PD-1/PD-L1 inhibitors. There was no difference in the incidence of irAEs among patients with different tumor types (3). Pillai et al. conducted a meta-

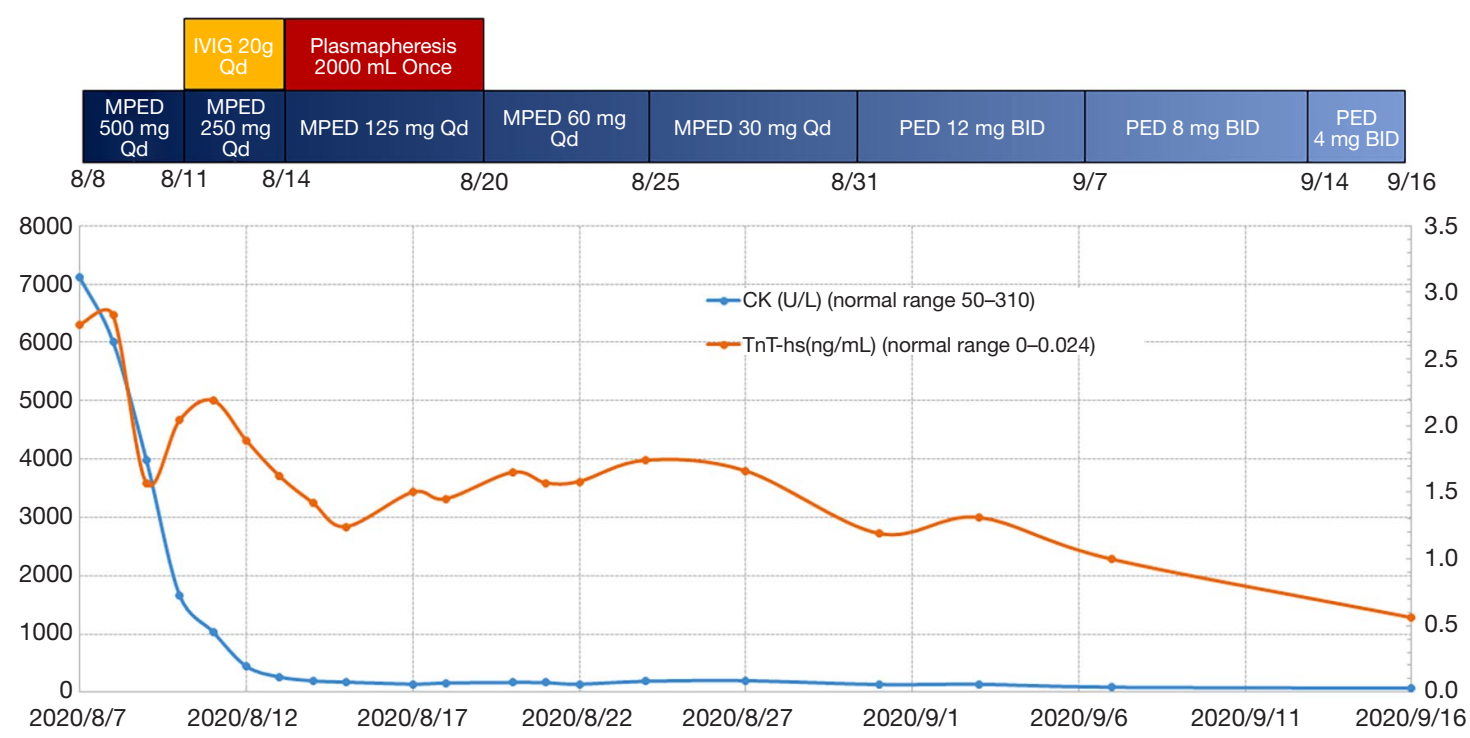

Figure 4 The trend in CK and TnT-hs with immunosuppressive therapy. CK, creatinine kinase; TnT-hs, high-sensitivity troponin T; MPED, methylprednisolone; PED, prednisolone. 
analysis of 5,744 patients with non-small cell lung cancer who underwent immunotherapy, and the results showed that fatigue was the most common type of adverse reaction. The incidence of all grades of irAEs with PD-1 inhibitors was slightly higher than that with PD-L1 inhibitors (16\% vs. $11 \%$ ), although the difference was not statistically significant (4). Wang et al. found that pneumonitis accounted for $35 \%$ of irAE-related fatalities, followed by hepatitis (22\%), enteritis (17\%), neurological events (15\%), and myocarditis $(8 \%)$. The median onset time of fatal irAEs was 40 days for a single-drug regimen and 14.5 days for a combination drug regimen. The median time from medication to death was 64 days for ipilimumab, 43 days for PD-1 inhibitors, and 35 days for PD-L1 inhibitors $(5,6)$.

The World Allergy Organization (WAO) classifies severe skin adverse reactions of drugs into SJS, TEN, SJS/ TEN, and drug hypersensitivity syndrome/drug reaction with eosinophilia and systemic symptoms (DRESS). SJS and TEN are different phenotypes in the spectrum of severe epidermolysis adverse reactions. They are immunemediated delayed-type hypersensitivity reactions with carry a high mortality. In addition to skin lesions, the clinical manifestations are often accompanied by visceral involvement and systemic poisoning symptoms. The main difference between the phenotypes is the degree of skin exfoliation: in SJS, exfoliation area is $<10 \%$ of the body surface area; in SJS/TEN the exfoliation area is $10-30 \%$ of the body surface area; and in TEN the exfoliation area is $>30 \%$ of the body surface area (7). The diagnosis is mainly based on clinical manifestations combined with pathological findings. The rash, which often affects the trunk, face, and palms, is characterized by extensive blistering on the erythematous skin and atypical target-like lesions. Most patients have erythema erosions of the oral cavity, genitals, and ocular mucosa and some of them may exhibit respiratory and gastrointestinal tract involvement. Skin biopsy pathology always reveals vesicles with extensive subcutaneous necrosis and keratinocyte apoptosis, and a small degree of lymphocytic infiltration. Bastuji-Garin et al. found that age $>40$ years old, heart rate $>120 \mathrm{bpm}$, a history of malignant tumors, an exfoliation area $>10 \%$, serum urea $>10 \mathrm{mmol} / \mathrm{L}$, serum bicarbonate $<20 \mathrm{mmol} / \mathrm{L}$, and blood sugar $>14 \mathrm{mmol} / \mathrm{L}$ were independent risk factors for a poor prognosis. Bastuji-Garin et al. established a severity-ofillness score for TEN(CCORTEN) to assess its severity and predict patient mortality (8). There is evidence to suggest that treatments such as glucocorticoids, intravenous immunoglobulin, cyclosporine $\mathrm{A}$, and plasmapheresis are effective (9). The case reported herein developed a relatively typical skin rash just 2 weeks after the infusion. His symptoms progressed rapidly and had a characteristic evolution process, which led to a clear diagnosis. From this case, it can be seen that severe skin rash, as the first irAE to appear, is an important indication of abnormal immune status and an early warning sign of subsequent multipleorgan involvement.

The incidence of severe myocarditis, the most lethal irAE, is about $0.09 \%(10)$, and the average mortality rate is $46 \%$. Onset usually occurs within 6 weeks after the first infusion. Some patients can develop severe myocarditis after only 1 or 2 treatment cycles. Anti-PD-1 therapy is associated with increased morbidity of myocarditis when it is combined with anti-CTLA-4 therapy, and the symptoms are more severe (11). ICI-related myocarditis can appear as various types of heart disease, including heart failure, various arrhythmias, myocardial pericarditis, cardiomyopathy, and sudden cardiac death. The ECG can show various arrhythmias, but the relative specificity is atrioventricular block. Approximately $90 \%$ of patients have troponin elevation, and in some patients, myoglobin, creatine kinase and its isoenzymes are increased. The continuous increases in various myocardial enzyme levels significantly exceed those in patients with myocardial infarction, and the recovery time is significantly prolonged. On echocardiography, severe left ventricular systolic dysfunction (LVEF <35\%) can be observed, which may be accompanied by changes in segmental wall motion and heart chamber enlargement (12). Because the clinical manifestations and examination results are similar to those of acute coronary syndromes, they need to be differentiated with the assistance of a cardiologist. Expert consensus recommends that patients with severe or critical myocarditis should be given intravenous methylprednisolone $(1 \mathrm{~g} /$ day $)$ for 3-5 days at first, and the dose should then be adjusted to $1-2 \mathrm{mg} / \mathrm{kg} / \mathrm{day}$ after the condition improves. The patient can be transitioned slowly to oral equivalent prednisone depending on their situation. The dose can be reduced every 1-2 weeks after the cardiac contraction and conduction function has been restored. The entire process should last for 6-8 weeks until the myocardial enzyme level recover to the baseline level. There is evidence to suggest that tacrolimus, antithymocyte globulin, infliximab, immunoglobulin, and plasmapheresis can be used as alternative treatments (12). In the case described herein, onset was sudden, the myocardial enzyme level increased sharply, the LVEF dropped rapidly to $30 \%$, 
and the ECG showed a characteristic change. After being transferred to the ICU, the patient received treatment to maintaining the balance of intake and output, along with antiplatelets, vasoactive drugs, hormone shock, IVIG, and plasmapheresis. After the treatments were adopted, various indicators improved, myocardial enzymes decreased, and the patient's physical strength gradually recovered. Hormone treatment lasted for nearly 3 months. As the most dangerous adverse event, myocarditis forms the core of the entire treatment process, and it is closely related to the patient's prognosis.

Inflammatory myopathy is the most common irAE affecting the nervous systems. Touat et al. reported that approximately $32 \%$ of ICI-related myositis is associated with myocarditis, and approximately $5 \%$ is associated with myasthenia gravis (13). Common symptoms include muscle soreness, muscle weakness of the proximal extremities, ptosis, eye movement weakness, labored speech/swallowing, and shortness of breath. Laboratory tests show elevated CK, and electromyography shows myogenic damage. The diagnosis is mainly based on muscle biopsy. Under an electron microscope, muscle fiber necrosis and inflammatory cell infiltration can be observed (14). The initial symptom in our patient was ptosis accompanied by weakness of the proximal extremities. However, he didn't exhibit the characteristics of lightness in the morning, heaviness in the evening, or improvement after rest. The respiratory muscles were also involved, and his symptoms progressed rapidly. CK showed a rapid upward trend; electromyography showed no neurogenic damage; acetylcholine receptor, acetylcholinesterase, titin, ryanodine receptor, and muscle-specific kinase antibodies were all negative; and the neostigmine test was also negative. The diagnosis of myasthenia gravis could be ruled out based on the existing evidence. After transfer to the ICU, the patient was continuously ventilated with a non-invasive ventilator, and his symptoms improved gradually with hormone therapy. No tracheal intubation or tracheotomy was required owing to the timely and effective treatment, which helped avoid secondary respiratory complications.

In summary, irAEs are not rare, and severe cases can be life-threatening. Severe irAEs appear early, progress rapidly, and involve multiple systems and organs, resulting in a high fatality rate. Early recognition and high-dose corticosteroids are the keys to successful treatment.

\section{Acknowledgments}

We thanked AME editing group for editing this manuscript.
Funding: This study was supported by the Beijing Tsinghua Changgung Hospital Fund (Grant No. 12017C1005, No. 12020C1005).

\section{Footnote}

Reporting Checklist: The authors have completed the CARE reporting checklist. Available at https://dx.doi. org/10.21037/tcr-21-470

Peer Review File: Available at https://dx.doi.org/10.21037/ tcr-21-470

Conflicts of Interest: All authors have completed the ICMJE uniform disclosure form (available at https://dx.doi. org/10.21037/tcr-21-470). The authors report the study was supported by the Beijing Tsinghua Changgung Hospital Fund (Grant No. 12017C1005, No. 12020C1005). The authors have no other conflicts of interest to declare.

Ethical Statement: The authors are accountable for all aspects of the work in ensuring that questions related to the accuracy or integrity of any part of the work are appropriately investigated and resolved. All procedures performed in studies involving human participants were in accordance with the ethical standards of the institutional and/or national research committee(s) and with the Helsinki Declaration (as revised in 2013). Written informed consent was obtained from the patient for publication of this case report and accompanying images. A copy of the written consent is available for review by the editorial office of this journal.

Open Access Statement: This is an Open Access article distributed in accordance with the Creative Commons Attribution-NonCommercial-NoDerivs 4.0 International License (CC BY-NC-ND 4.0), which permits the noncommercial replication and distribution of the article with the strict proviso that no changes or edits are made and the original work is properly cited (including links to both the formal publication through the relevant DOI and the license). See: https://creativecommons.org/licenses/by-nc-nd/4.0/.

\section{References}

1. Reck M, Rodríguez-Abreu D, Robinson AG, et al. Pembrolizumab versus Chemotherapy for PD-L1Positive Non-Small-Cell Lung Cancer. N Engl J Med 
2016;375:1823-33.

2. Wang PF, Chen Y, Song SY, et al. Immune-Related Adverse Events Associated with Anti-PD-1/PD-L1 Treatment for Malignancies: A Meta-Analysis. Front Pharmacol 2017;8:730.

3. De Velasco G, Je Y, Bossé D, et al. Comprehensive Metaanalysis of Key Immune-Related Adverse Events from CTLA-4 and PD-1/PD-L1 Inhibitors in Cancer Patients. Cancer Immunol Res 2017;5:312-8.

4. Pillai RN, Behera M, Owonikoko TK, et al. Comparison of the toxicity profile of PD-1 versus PD-L1 inhibitors in non-small cell lung cancer: A systematic analysis of the literature. Cancer 2018;124:271-7.

5. Wang DY, Salem JE, Cohen JV, et al. Fatal Toxic Effects Associated With Immune Checkpoint Inhibitors: A Systematic Review and Meta-analysis. JAMA Oncol 2018;4:1721-8.

6. Thompson JA, Schneider BJ, Brahmer J, et al. NCCN Guidelines Insights: Management of ImmunotherapyRelated Toxicities, Version 1.2020. J Natl Compr Canc Netw 2020;18:230-41.

7. Maloney NJ, Ravi V, Cheng K, et al. Stevens-Johnson syndrome and toxic epidermal necrolysis-like reactions to checkpoint inhibitors: a systematic review. Int J Dermatol

Cite this article as: Cao J, Li Q, Zhi X, Yang F, Zhu W, Zhou T, Hou X, Chen D. Pembrolizumab-induced autoimmune Stevens-Johnson syndrome/toxic epidermal necrolysis with myositis and myocarditis in a patient with esophagogastric junction carcinoma: a case report. Transl Cancer Res 2021;10(8):3870-3876. doi: 10.21037/tcr-21-470 2020;59:e183-8.

8. Bastuji-Garin S, Fouchard N, Bertocchi M, et al. SCORTEN: a severity-of-illness score for toxic epidermal necrolysis. J Invest Dermatol 2000;115:149-53.

9. Creamer D, Walsh SA, Dziewulski P, et al. U.K. guidelines for the management of Stevens-Johnson syndrome/ toxic epidermal necrolysis in adults 2016. Br J Dermatol 2016;174:1194-227.

10. Johnson DB, Balko JM, Compton ML, et al. Fulminant Myocarditis with Combination Immune Checkpoint Blockade. N Engl J Med 2016;375:1749-55.

11. Moslehi JJ, Salem JE, Sosman JA, et al. Increased reporting of fatal immune checkpoint inhibitor-associated myocarditis. Lancet 2018;391:933.

12. Mahmood SS, Fradley MG, Cohen JV, et al. Myocarditis in Patients Treated With Immune Checkpoint Inhibitors. J Am Coll Cardiol 2018;71:1755-64.

13. Touat M, Maisonobe T, Knauss S, et al. Immune checkpoint inhibitor-related myositis and myocarditis in patients with cancer. Neurology 2018;91:e985-94.

14. Selva-O'Callaghan A, Pinal-Fernandez I, TralleroAraguás E, et al. Classification and management of adult inflammatory myopathies. Lancet Neurol 2018;17:816-28. 